perform some sampling of a population of Pearson's Type I. As the method used is a simple one and apparently new, and appears to give satisfactory results, a description of it may be of interest.

A stout cardboard disc was accurately subdivided into a number of sectors, such that the angle of each sector was proportional to the number of individuals of the smoothed population corresponding to each of the equal steps of the independent variable. The disc was then pinned down by its centre on top of a cardboard washer and spun rapidly. While still spinning so fast that the markings were indistinguishable, it was stabbed with a sharp instrument and brought to rest. The value attached to the stabbed sector was then noted.

As to the rate of sampling, once the disc is made up, at least 500 samples can be taken in this way per hour per person, using the left hand for spinning and stabbing.

Chas. E. R. Bruce.

W. Bevan Whitney.

The British Electrical and Allied Industries Research Association,

36 and 38 Kingsway, London, W.C.2, May 12.

\section{The Occurrence of Indium in Tin.}

Mr. M. W. Garretr has recently reported the general occurrence of traces of indium in tin (NATURE, Feb. 12, 1927, p. 260). I wish to confirm this observation, which was also made here last January in the course of some other work.

Indium was then detected spectrographically in three samples of tin, and of a number from varying sources since examined only four were found free from it. These included two specimens containing 98.94 per cent. and 97.60 per cent. tin, probably of Chinese origin, the leady ' $\mathrm{RBW}$ ' brand from electrolytic detinning of tinplate scrap, and the extremely pure 'Chempur' brand (99:998 + per cent. tin). All 'English' brands tested showed strong traces of indium.

Several varieties of soft solder gave a positive result also, and in both tin and solder the most persistent line was found to be $\lambda=4102$, the next in persistency being $\lambda=4511$, in agreement with Garrett's result in the case of tin, and contrary, as he has remarked, to de Gramont's.

Indium does not appear to be recognised at all as an impurity in tin. The almost complete neglect of spectral methods of analysis by metallurgists and others may explain this, as the line at 4511 is close to the strong tin line at 4525 , and can scarcely fail to be noticed if present.

J. R. GREEN.

The Laboratory,

Batchelor, Robinson and Co., Ltd.,

Nevill's Dock, Llanelly,

May 13.

\section{Sand-files and Kala-azar.}

IN the issue of Nature dated Mar. 26, p. 460, there is a letter from the Kala-azar Commission of the Royal Society working in north China in which the claim is made that for the first time an infection with kala-azar has been produced by means of the intraperitoneal inoculation of the midgut contents of the genus Phlebotomus into an experimental animal.

As the letter is dated Jan. 28 the mistake is a natural one, since the writers cannot then have seen the report of a similar and earlier experimental success on the same lines obtained by the Indian Kala-azar Commission in Assam. The report of this experiment, in which a white mouse was infected with kala-azar by the intra-peritoneal inoculation of the alimentary canal contents of $P$. argentipes infected by feeding on kala-azar cases, was published in the Jan. 1927 number of the Indian Journal of Medical Research, but was actually received for publication on Sept. 6,1926 . The fact that the workers in China were working with $P$. sergenti var. and the hamster Cricetulus griseus, while the Indian Commission was working with $P$. argentipes and the white mouse, does not affect the question of priority at issue.

H. E. Short.

Kala-azar Commission, . (Director.)

Shillong, Assam, India, May 14.

\section{Early Suggestion as to the Vacuum Flask.}

THE following extract from "Mechanical Inventions and Suggestions," by Lewis Gompertz, may interest some readers of NATURE :

"Suggestion to produce a Fire-Proof Box.

"That this suggestion would succeed, I am far from trusting to, but as possibly it might to some extent do so, it may not $b \dot{\theta}$ amiss to describe it untried. The box is made of steel, etc., highly polished, as polished metals reflect back the heat as well as the light that are cast upon them. This box is then put into a much larger air-tight case of iron, and has legs of thin wire to prevent it touching the outside case, and all the air is pumped out so as to leave a vacuum between the two boxes, and so that no heat can be conveyed to it by the contact of air ; and as the radiant heat is rendered harmless by the polish, and the heat of contact is prevented by the vacuum, it seems that the heat is kept out entirely, excepting through the small wire legs."

The book, which is a second edition, unfortunately bears no printed date, but was given to me in $\mathbf{1 8 5 6}$. Lewis Gompertz was secretary to the Animals' Friend Society about 1840 .

6 Croftdown Road, London, N.W.5,

RtChaRd Inwards. May 15.

\section{The Hythe Skulls.}

IN a recent number of Biometrika (vol. 18, p. 22) Miss Hooke writes that the measurements which I made upon the well-known skulls at Hythe were " made on 590 crania selected from at least double that number." The impression which this gives, and actually has given, is that, out of a large number, half had been selected or chosen for some special reason, and that, therefore, probably they did not fairly represent the whole collection. The skulls were in no way 'selected,' but I had measured all which were available at the time, though more have since been recovered.

Miss Hooke says that she believes that these were skulls of Kentish people, while the whole of my work shows that they were those of brachycephalic immigrants of the Alpine race who had settled in Hythe, probably quite peacefully, in or before the fourteenth century.

The work on the Hythe skulls has been so often quoted by anthropologists that I feel that I must correct what I regard as a serious mis-statement.

St. Thomas's Hospital, F. G. Parsons. 